\title{
Analysis of conditional heterozygous STXBP1 mutations in human neurons
}

\author{
Christopher Patzke, ${ }^{1}$ Yan Han, ${ }^{1}$ Jason Covy, ${ }^{1}$ Fei Yi, ${ }^{1}$ Stephan Maxeiner, ${ }^{1}$ Marius Wernig, ${ }^{2}$ and Thomas C. Südhof ${ }^{1,3}$ \\ 'Department of Molecular and Cellular Physiology, ${ }^{2}$ Institute for Stem Cell Biology and Regenerative Medicine and Department of Pathology, and ${ }^{3}$ Howard Hughes Medical Institute, Stanford University \\ School of Medicine, Stanford, California, USA
}

\begin{abstract}
Heterozygous mutations in the syntaxin-binding protein 1 (STXBP1) gene, which encodes Munc18-1, a core component of the presynaptic membrane-fusion machinery, cause infantile early epileptic encephalopathy (Ohtahara syndrome), but it is unclear how a partial loss of Munc18-1 produces this severe clinical presentation. Here, we generated human ES cells designed to conditionally express heterozygous and homozygous STXBP1 loss-of-function mutations and studied isogenic WT and STXBP1-mutant human neurons derived from these conditionally mutant ES cells. We demonstrated that heterozygous STXBP1 mutations lower the levels of Munc18-1 protein and its binding partner, the t-SNARE-protein Syntaxin-1, by approximately $30 \%$ and decrease spontaneous and evoked neurotransmitter release by nearly $50 \%$. Thus, our results confirm that using engineered human embryonic stem (ES) cells is a viable approach to studying disease-associated mutations in human neurons on a controlled genetic background, demonstrate that partial STXBP1 loss of function robustly impairs neurotransmitter release in human neurons, and suggest that heterozygous STXBP1 mutations cause early epileptic encephalopathy specifically through a presynaptic impairment.
\end{abstract}

\section{Introduction}

Recent advances in human genetics identified hundreds of heterozygous mutations that may cause neurodevelopmental disorders such as mental retardation, autism, or schizophrenia (1-5). The identification of these mutations raises 2 major questions: is a given mutation truly pathogenic, or does it represent a polymorphism? If a mutation is pathogenic, how does it produce impairments that lead to disease? A traditional approach to addressing these questions is to study neurons differentiated from patient-derived induced pluripotent stem (iPS) cells compared with neurons derived from control iPS cells to identify potential abnormalities (6-17). Although powerful, this approach does not necessarily reveal whether a mutation produces a particular phenotype because the test and control neurons analyzed carry different genetic backgrounds and are derived from distinct iPS cell clones $(18,19)$. Genetic backgrounds are potentially important, since many disease-associated mutations can clinically produce distinct phenotypes.

Hundreds of heterozygous mutations in the syntaxin-binding protein 1 (STXBP1) gene have been observed in patients with severe forms of early epileptic encephalopathy (referred to as Ohtahara or as West syndrome; refs. 20-22) and other, usually severe, clinical presentations (23-25). However, it is unknown how the STXBP1 mutations and a partial loss of Munc18-1 affect human neural function, whether these mutations produce disease by causing a nonneuronal impairment, and whether the changes produced by $S T X B P 1$ mutations are potentially amenable to therapy $(26,27)$.

Authorship note: Christopher Patzke and Yan Han contributed equally to this work Conflict of interest: The authors have declared that no conflict of interest exists. Submitted: August 19, 2014; Accepted: June 11, 2015.

Reference information: J Clin Invest. 2015;125(9):3560-3571. doi:10.1172/JCI78612.

\section{Results}

In order to address these questions, we need an approach that allows us to test how heterozygous loss of function of STXBP1 specifically affects human neuronal properties in cells with a controlled genetic background. To this end, we used homologous recombination to mutagenize the STXBP1 gene encoding Munc18-1 in human H1 embryonic stem (ES) cells (Figure 1A). ES cells (which do not express Munc18-1) were infected with a recombinant adeno-associated virus (AAV) that contains WT human STXBP1 sequences from the region encoding exon 2. In the AAV, exon 2 was flanked by loxP sites (for deletion of the exon by Cre-recombinase), and a drug selection resistance cassette that is surrounded by frt sites (for deletion by Flprecombinase) was additionally inserted adjacent to the $5^{\prime}$ loxP site (Figure 1A). AAVs with 2 different resistance markers were produced to allow generation of hetero- and homozygous conditional KO (cKO) cells. Multiple drug-resistant clones were isolated and screened by PCR. Two independent heterozygous and homozygous ES cell clones were selected for analyses (Figure 1B and Supplemental Figure 1, A and C; supplemental material available online with this article; doi:10.1172/JCI78612DS1). This cKO approach was designed to allow analysis of the effects of hetero- and homozygous mutations in human cells on a controlled genetic background, thereby eliminating potentially confounding effects induced by genetic background changes or selection of cell clones (28).

To analyze the phenotypic consequences of STXBP1 loss-offunction mutations, we used the induced neuron (iN cell) approach in which neurons are produced from ES or iPS cells by forced expression of transcription factors $(29,30)$. We first tested whether heterozygous and homozygous STXBP1-mutant ES cells could be efficiently 
A
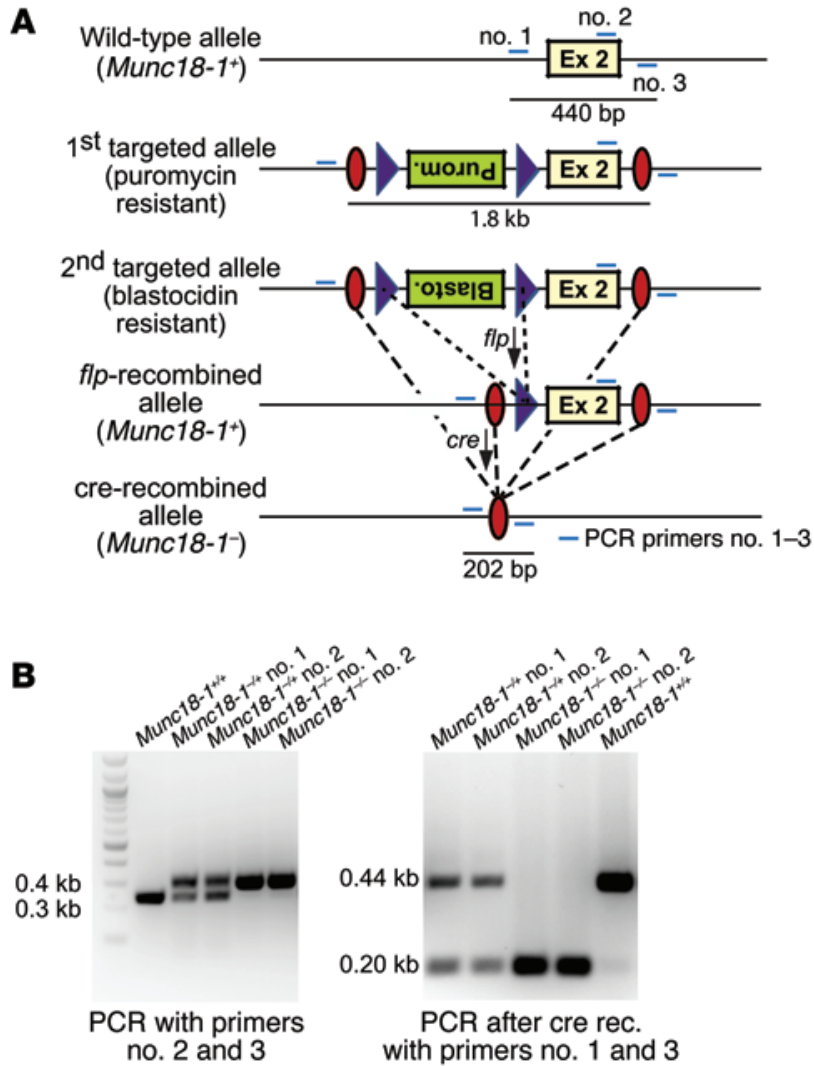

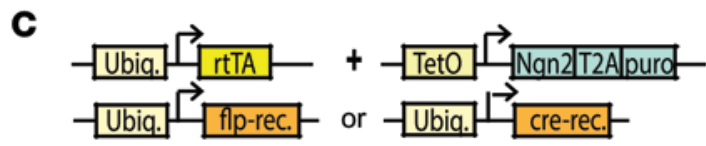

D

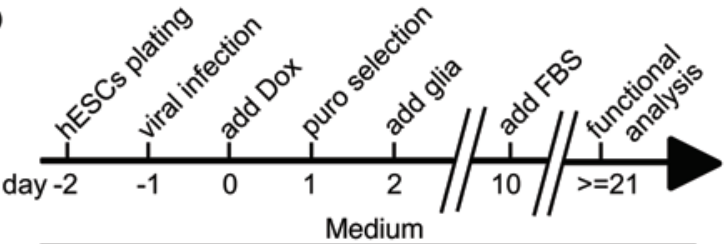

\begin{tabular}{l|l|l|l|}
\hline mTeSR & N2 & NB+B27 & NB+B27+FBS \\
\hline
\end{tabular}

\section{$\mathbf{E}$}
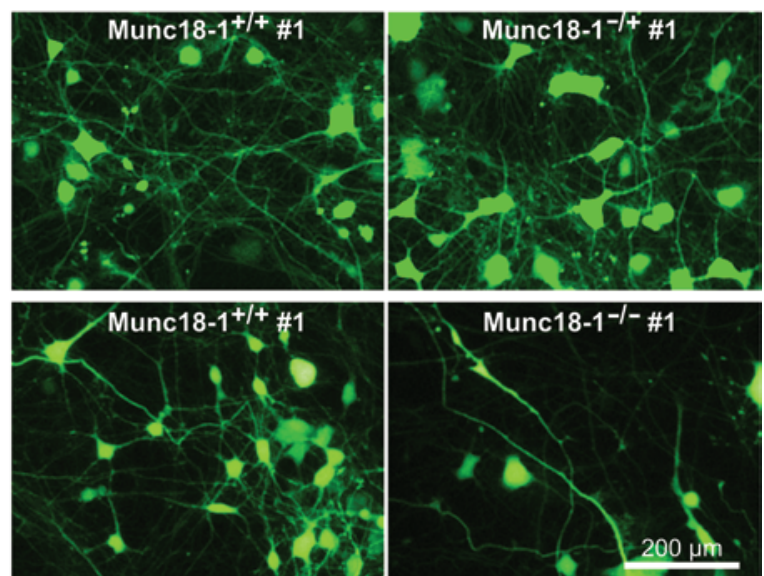

Figure 1. Genetic engineering of conditional STXBP1 gene mutations in human ES cells and generation of iN cells from conditionally mutant ES cells. (A) Targeting strategy. The STXBP1 gene was mutated by homologous recombination in H1 ES cells using AAVs containing the indicated sequences. Drug-resistant clones were confirmed by PCR using the primers no. 1 to no. 3. Ex 2, exon 2; red ovals, loxP sites; blue triangles, frt sites. (B) PCR analysis of WT ES cells and 2 independent heterozygous and homozygous ES cell clones. PCRs were performed with the indicated primers (see $\mathbf{A}$ ). In this panel, Munc18-1+/+ refers to untargeted ES cells. (C) Design of lentiviral vectors for rapid Ngn2-mediated directed differentiation of ES cells into iN cells. (D) Flow diagram of iN cell experiments. Conditionally mutant ES cells were coinfected at day -1 with the lentiviruses used for iN cell generation (shown in C) plus a lentivirus expressing either Flp-recombinase (which removes the resistance cassette and reactivates STXBP1 expression, resulting in Munc18-1+/+ neurons) or Cre-recombinase (which deletes exon 2 of the STXBP1 gene, resulting in Munc18-1//+ or Munc18-1/- neurons). (E) Representative fluorescence images of control and mutant iN cells derived from heterozygous (top) or homozygous conditionally STXBP1-mutant ES cells (bottom). ES cells were coinfected at the day of iN cell induction with an EGFP-expressing lentivirus for visualizing neurons; pictures were taken at day 23 . Scale bar: $200 \mu \mathrm{m}$. For additional data on the selection of neurons and more representative images, see Supplemental Figure 1.

converted into iN cell neurons by expression of the transcription factor Neurogenin-2 (Ngn2) using a variation of our standard protocol described earlier (Figure 1, C and D, and ref. 29). We coinfected ES cells at day -1 with viruses expressing Ngn2 and reverse tetracyclinecontrolled transactivator (rtTA) (which drives directed differentiation of the ES cells into neurons by the standard procedure, ref. 29) and additionally with viruses expressing either Flp-recombinase (to remove the resistance cassette and create an active $S T X B P 1$ allele) or Cre-recombinase (to remove exon 2 and inactivate $S T X B P 1$ expression, since deletion of exon 2 creates a premature stop codon). As a result, we produced from the same population of ES cells isogenic WT control neurons (referred to as Munc18-1+/+) and heterozygous or homozygous STXBP1-mutant neurons (referred to as $M u n c 18-1^{-/+}$or Munc18-1/-, respectively). Production of these neurons was similarly efficient with coexpression of Flp- or Cre-recombinases. However, we noticed that homozygous but not heterozygous STXBP1-mutant Munc18-1/- neurons started to degenerate after 1 week and showed massive neuronal cell death during 3 weeks in culture (Figure 1E and Supplemental Figure 3A; see analysis below).
Immunoblotting analysis revealed that heterozygous STXBP1mutant neurons exhibited a decrease in Munc18-1 protein levels, whereas homozygous STXBP1-mutant neurons lacked Munc18-1 protein, as expected (Figure 2A). Quantification of the levels of Munc18-1 protein in iN cells derived from 2 independent heterozygous STXBP1-mutant ES cell clones demonstrated that both were subject to a similar decrease in Munc18-1 levels ( 30\% decrease; Figure 2B and Supplemental Figure 1B for mRNA levels). A survey of other synaptic proteins revealed that Syntaxin-1 levels were also selectively decreased (Figure 2B and Supplemental Figure 2, A and B). No other protein examined showed changes in levels. These results agree with studies in mice in which Syntaxin-1 protein levels are suppressed when Munc18-1 protein is deleted and Munc18-1 protein levels are conversely suppressed when Syntaxin-1 expression is ablated (31-34), suggesting that Munc18-1 and Syntaxin-1 behave like interdependent subunits of a complex that stabilize each other.

Since homozygous STXBP1-mutant neurons appeared to degenerate (Figure $1 \mathrm{E}$ ), we measured the survival of neurons with heterozygous or homozygous STXBP1 mutations as a function of 
A
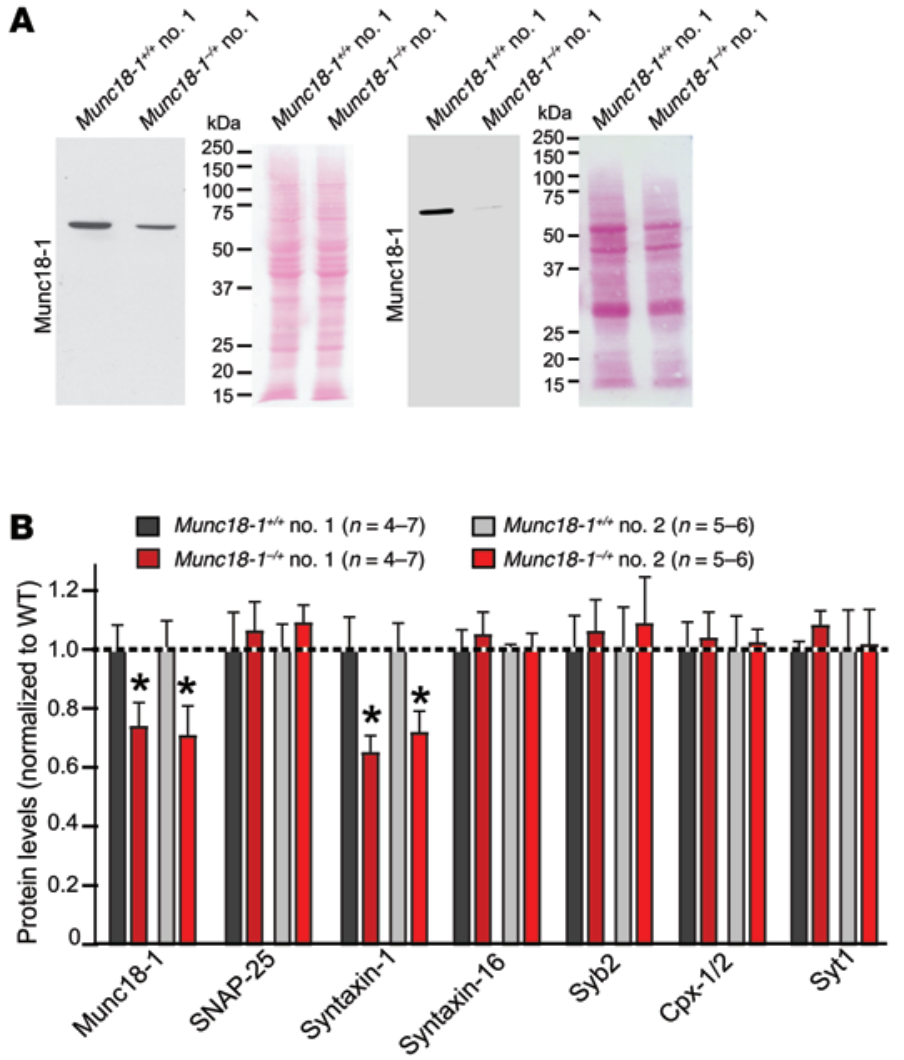

C

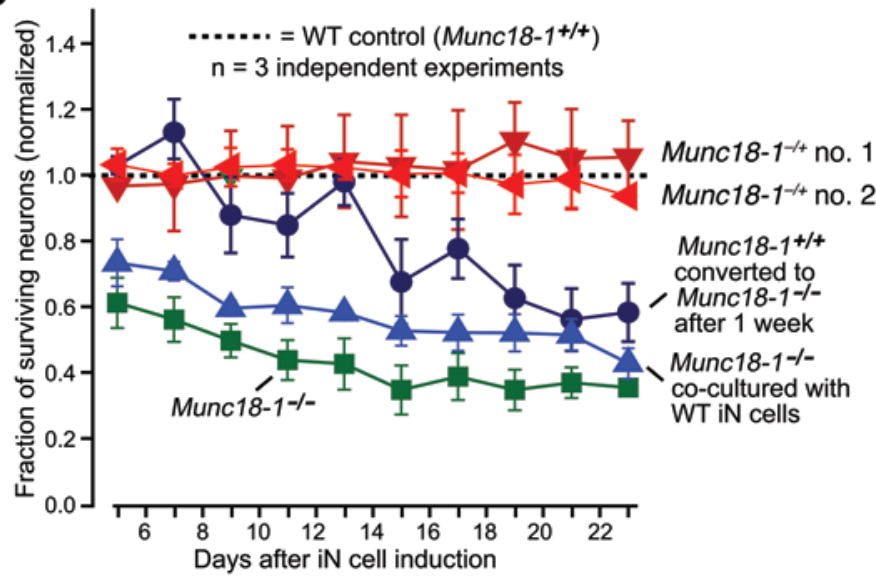

D

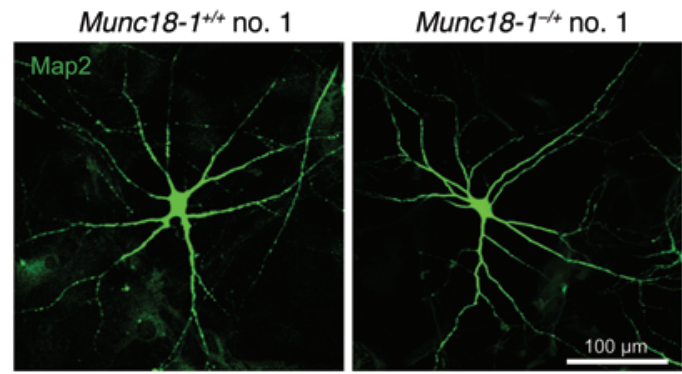

E

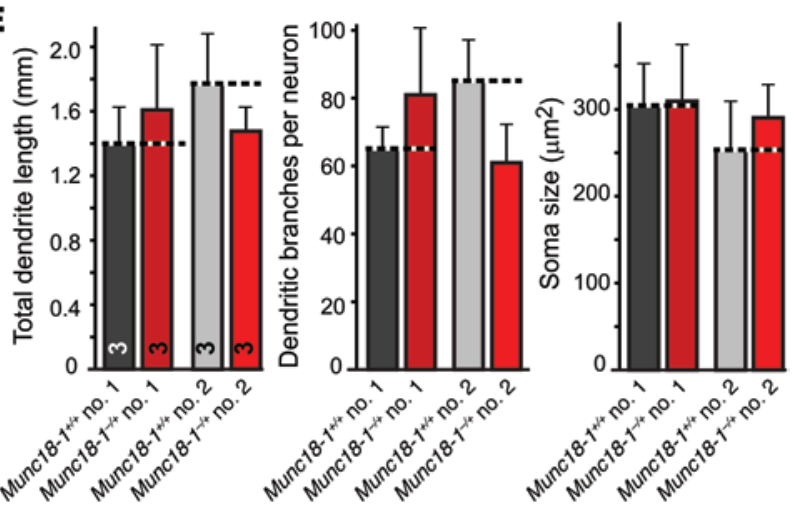

F

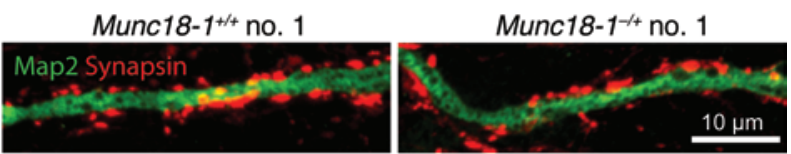

G
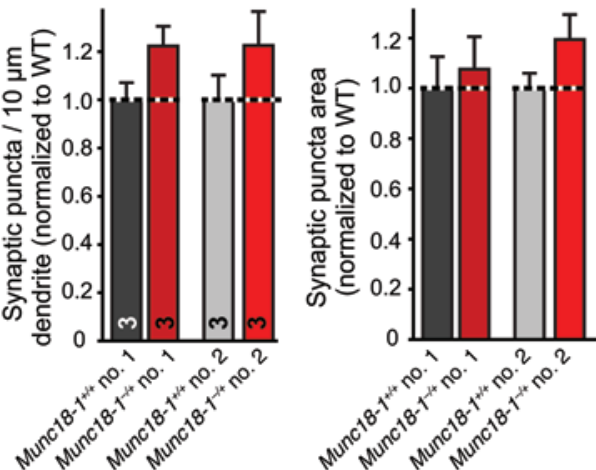

Figure 2. Protein composition, survival, and neuronal differentiation of STXBP1-mutant human neurons. (A) Immunoblots and Ponceau-stained blots of control and heterozygous and homozygous mutant iN cells. (B) Protein levels in matched control and independent clones of heterozygous STXBP1-mutant iN cells, determined by quantitative immunoblotting (see also Supplemental Figure 2 , A and B). ${ }^{*} P<0.05$, Student's $t$ test. (C) Plot of the fraction of surviving neurons compared with controls (dotted line) as a function of culture time. Tested conditions: heterozygous cells from 2 independent ES cell clones (red); homozygous cells generated with standard conditions cultured alone (green) or cocultured with WT iN cells (blue); homozygous STXBP1-mutant iN cells in which the mutation was induced 1 week after iN cell induction (black). Degeneration of homozygous STXBP1-mutant neurons is statistically highly significant under all conditions. $P<0.01,2$-way ANOVA. (D) Heterozygous STXBP1-mutant iN cells stained for the dendritic marker MAP2. Scale bar: 100 4m. (E) Total dendritic length (left), number of branches (middle), and soma size (right) quantified with control and mutant iN cells derived from 2 separate mutant ES cell clones. (F) Dendrites from control and heterozygous STXBP1-mutant iN cells stained for MAP2 and synapsin to visualize presynaptic terminals. Scale bar: $10 \mu \mathrm{m}$. (G) Density (left) and size (right) of synapsin-positive puncta along dendrites in heterozygous STXBP1-mutant iN cells derived from 2 independent ES cell clones. Error bars represent mean \pm SEM. Numbers of independent experiments performed are indicated in the graphs.

time in culture (Figure 2C and Supplemental Figure 3A). We found that heterozygous STXBP1-mutant neurons displayed no difference in survival compared with control neurons, whereas homozygous STXBP1-mutant neurons already exhibited a relative loss of neurons $(\sim 40 \%)$ at the first time point examined (day 5 after iN cell induction). The homozygous STXBP1-mutant neurons continued to die over time. Homozygous STXBP1-mutant neurons also degenerated when cocultured with WT human neurons, indicating that the degeneration was cell autonomous (Figure 2C). This neurodegeneration phenotype is consistent with observations in con- 

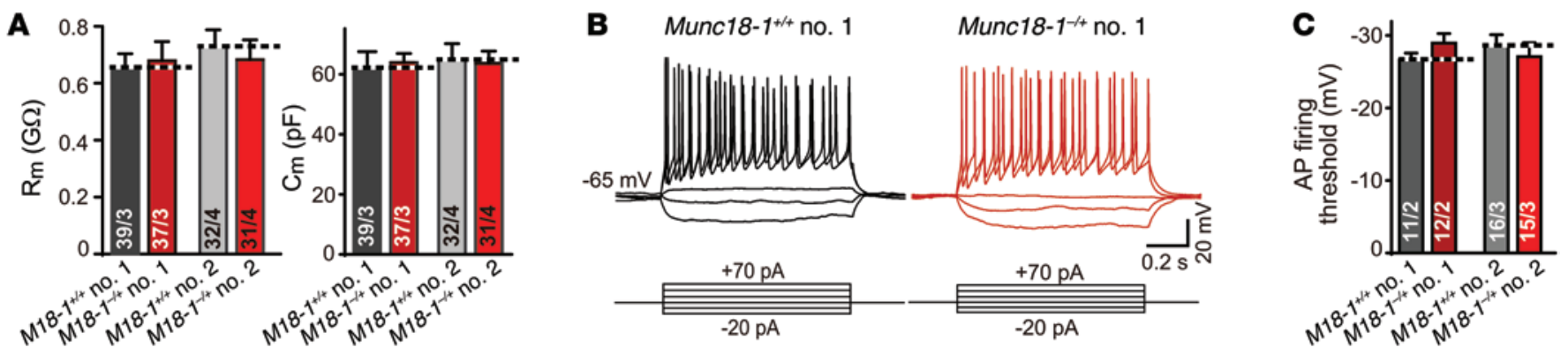

Figure 3. Normal intrinsic electrical properties in heterozygous STXBP1-mutant neurons. (A) Heterozygous STXBP1-mutant iN cells exhibit no changes in input resistance (left) or capacitance (right). Neurons derived from 2 different mutant ES cell clones were analyzed as indicated. (B) Representative traces of analyses of the action potential firing properties of control and heterozygous STXBP1-mutant iN cells. Neurons held in current-clamp mode were injected with increasing current pulses (10 pA increments). Experimental protocol is shown at the bottom. (C) Summary graph of the action potential firing thresholds determined in control and heterozygous mutant iN cells derived from 2 different ES cell clones. Summary graphs show mean \pm SEM; numbers of cells/independent cultures analyzed are indicated in the bars.

stitutive Munc18-1-KO mice (31). Moreover, when we introduced the homozygous STXBP1 deletion after iN cells had already been generated (1 week after iN cell induction), the neurons still rapidly degenerated, demonstrating that Munc18-1 protein is required for neuronal survival rather than neuron specification (Figure 2C). In a second independent approach assessing the survival of homozygous STXBP1-mutant neurons, we measured the relative amount of human GAPDH genomic DNA and mRNA in WT and Munc18-1depleted iN cells after 4 weeks of culture. The mutant neurons exhibited approximately $70 \%$ and $60 \%$ cell death, respectively, based on their GAPDH DNA or mRNA levels (Supplemental Figure $3, \mathrm{~B}$ and $\mathrm{C}$ ). Because of their degeneration, no further studies were performed on homozygous STXBP1-mutant neurons.

We next tested to determine whether heterozygous STXBP1mutant neurons were similar to WT neurons in terms of shape, dendritic arborization, and synapse numbers. Quantifications of the total length of dendrites and of the number of dendritic branches per neuron as well as of their soma size detected no changes (Figure 2, D and E). Similarly, measurements of the number of synapses per dendritic segment or the synapse sizes also failed to identify differences between WT and STXBP1-mutant neurons (Figure 2, F and G). Thus, the heterozygous $S T X B P 1$ mutation does not impede the normal development of a neuron or synaptogenesis.

Led by the hypothesis that human neurons with a heterozygous STXBP1 mutation may exhibit functional changes, we analyzed the neurons electrophysiologically. Consistent with the absence of morphological changes, we detected no alterations in intrinsic electrical properties (Figure 3, A-C). Measurements of spontaneous miniature excitatory postsynaptic currents (mEPSCs), however, revealed the same phenotype in neurons derived from both independent mutant ES cell clones: a large decrease in mEPSC frequency without a change in MEPSC amplitude, indicative of an impairment in presynaptic neurotransmitter release (Figure 4, A and B). Cumulative distribution plots of interevent
A

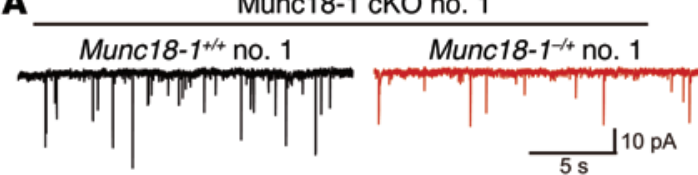

mEPSC frequency

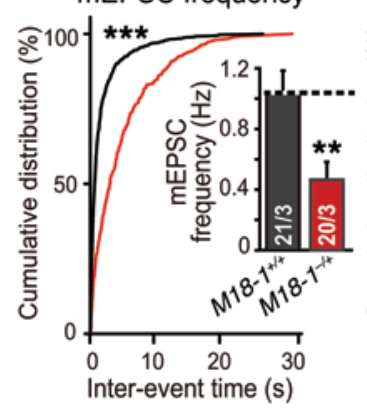

mEPSC amplitude

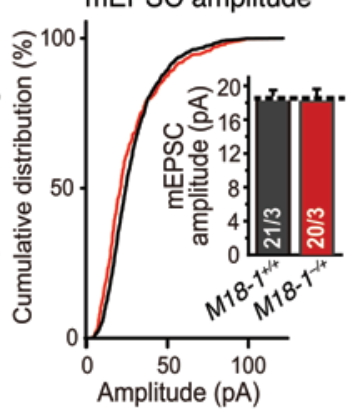

B

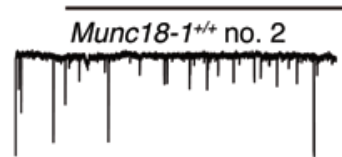

mEPSC frequency

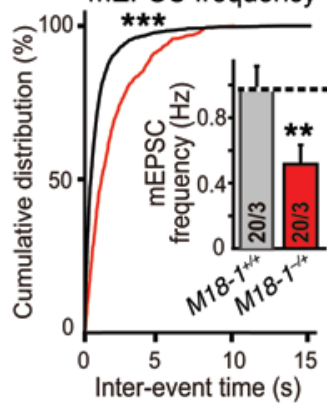

Munc18-1 cKO no. 2
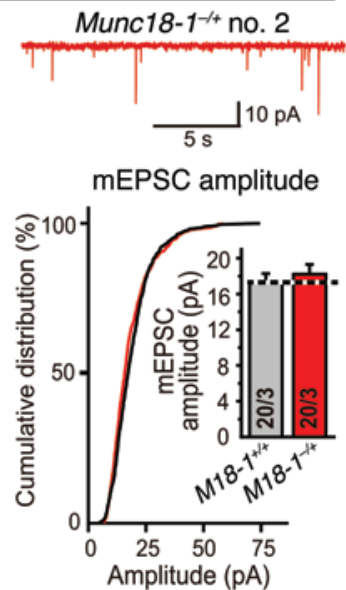

Figure 4. Decreased spontaneous neurotransmitter release in heterozygous STXBP1-mutant human neurons. (A and B) Impaired spontaneous neurotransmitter release in heterozygous STXBP1-mutant human neurons. Representative traces of mEPSCs recorded in $1 \mu \mathrm{M}$ tetrodotoxin (TTX) from 2 different clones are shown on top. Summary graphs of the mEPSC parameters are shown below. Left, cumulative plot of the mEPSC interevent interval (inset: mean mEPSC frequency); right, cumulative plot of the mEPSC amplitude (inset: mean mini-amplitude). ${ }^{* *} P<0.01$, unpaired, 1 -tailed Student's $t$ test for comparison of the means; ${ }^{* *} P<0.001$, Kolmogorov-Smirnov test for comparison of cumulative distributions. Summary graphs exhibit mean \pm SEM; numbers of cells/independent cultures analyzed are indicated in the bars. 
A

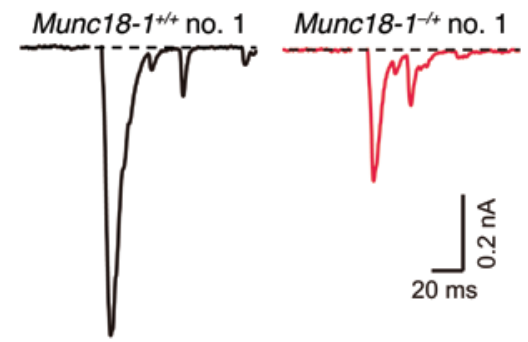

B

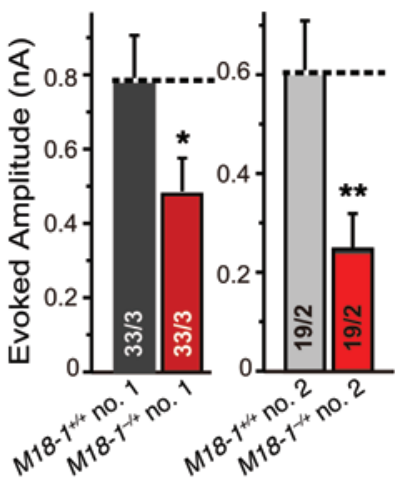

C

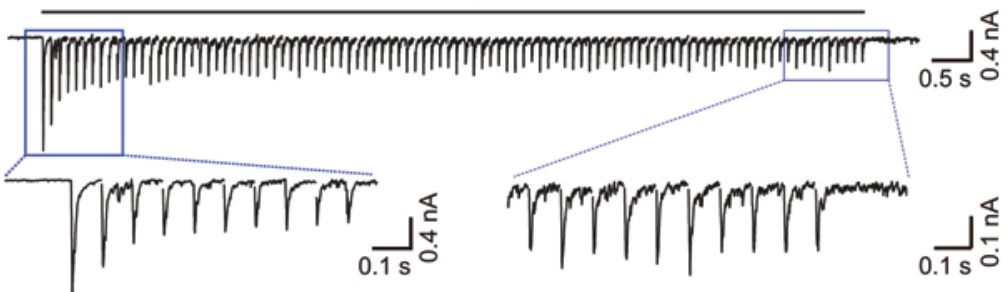

Munc18-1-1/4 no. 1

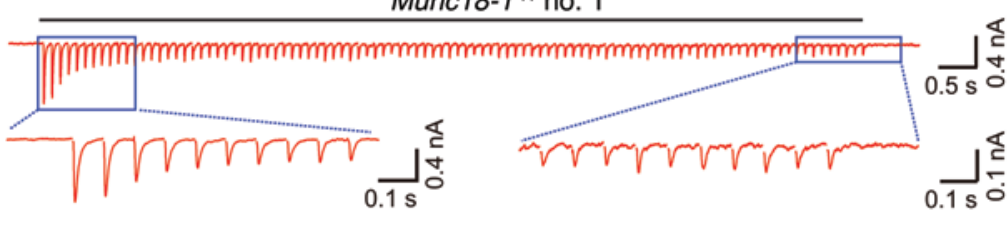

D

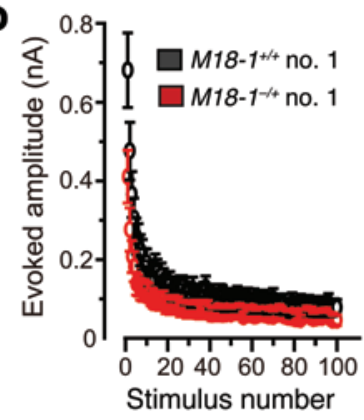

E

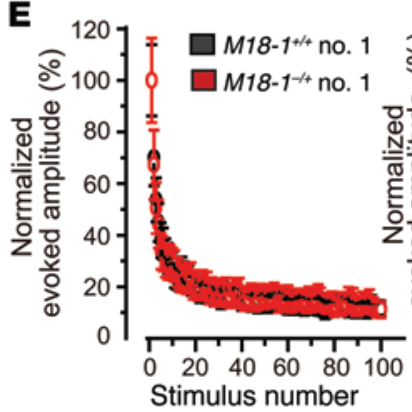

F Munc18-1+1/+ no. 1 Sucrose

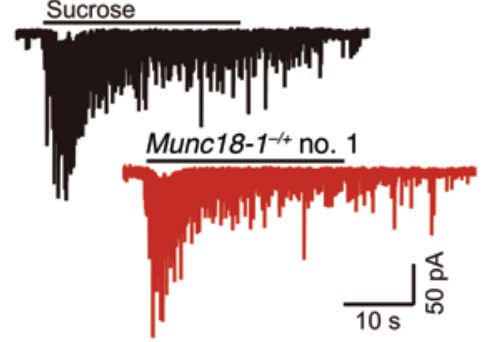

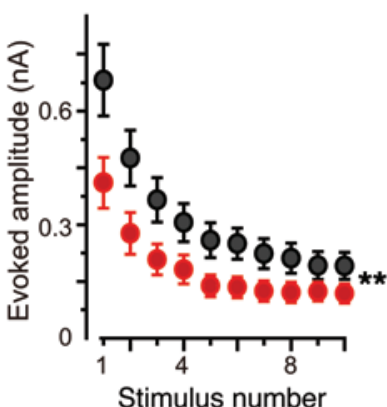

Stimulus number

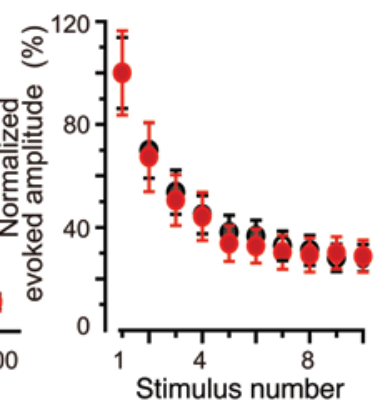

G

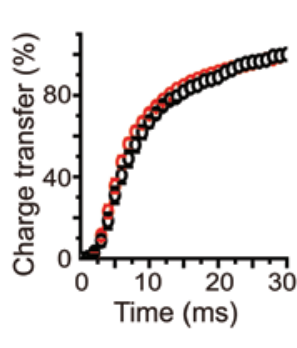

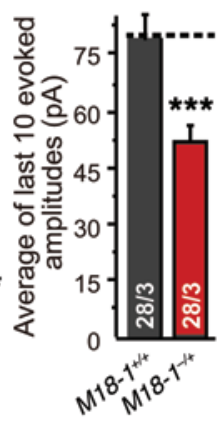
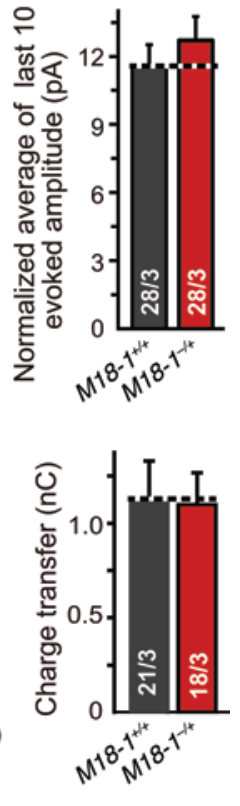

Figure 5. Heterozygous STXBP1 mutations impair evoked neurotransmitter release. (A and $\mathbf{B}$ ) Representative traces (A) and amplitude summary graphs (B) of EPSCs evoked by isolated action potentials in control and STXBP1-mutant neurons derived from 2 different ES cell clones. (C) Representative traces of EPSCs evoked by $10 \mathrm{~Hz} / 10$ second stimulus trains in control and heterozygous STXBP1-mutant human neurons. ( $\mathbf{D}$ and $\mathbf{E}$ ) Quantitative analyses of EPSCs evoked by $10 \mathrm{~Hz}$ stimulus trains as absolute (D) or relative amplitudes normalized to the first response (E). The amplitudes over the entire 10-second train (left panels) and over the first 10 stimuli (middle panels) are plotted as a function of stimulus number, while the average amplitudes evoked by the last 10 stimuli are shown in the right panels. Note that while heterozygous STXBP1-mutant neurons exhibit uniformly reduced absolute amplitudes, synaptic plasticity as reflected by relative amplitudes is normal. ( $\mathbf{F}$ and $\mathbf{G}$ ) Measurements of release induced by hypertonic sucrose to assess the size of the RRP of vesicles. Panels show representative traces (F) and summary graphs of the cumulative charge transfer as a function of time (G, left) or of the total mean charge transfer (G, right). Summary graphs show mean \pm SEM; numbers of cells/independent cultures analyzed are indicated in the bars. Statistical comparisons were made by Student's $t$ test comparing heterozygous STXBP1 mutants to controls. ${ }^{*} P<0.05$; ${ }^{* *} P<0.01 ;{ }^{* *} P<0.001$.

times demonstrated that this impairment was uniformly distributed across synapses, i.e., that there was a very large shift to lower $\mathrm{mEPSC}$ frequencies in all synapses (Figure 4, A and B).

To further characterize synaptic transmission in Munc18-1 mutant neurons, we examined evoked synaptic responses. We observed in neurons derived from both mutant ES cell clones that heterozygous STXBP1 mutations caused a dramatic decrease $(\sim 45 \%)$ in action potential-evoked EPSCs similar to the change in $\mathrm{mEP}$ SC frequency (Figure 5, A and B). The amplitudes of EPSCs evoked by a $10 \mathrm{~Hz} / 10$ second stimulus train were also decreased (Figure 5, C and D). EPSC amplitudes were uniformly decreased throughout the $10 \mathrm{~Hz}$ train in absolute terms (Figure 5D), but analyses of the relative EPSCs normalized to the first response detected no differences between control and STXBP1-mutant neurons (Figure $5 \mathrm{E}$ ), demonstrating that short-term synaptic plasticity was unchanged.

The observed phenotype could potentially be accounted for by, among other factors, a decrease in synapse numbers or a decrease in the size of the readily releasable pool (RRP) of vesicles per synapse. Our morphological analysis above showed that synapse numbers were unchanged in heterozygous STXBP1mutant neurons, ruling out the first possibility (Figure 2, F and G). We thus measured the size of the RRP, using stimulation by hypertonic sucrose as a tool to estimate the RRP (Figure 5F and ref. 35). Surprisingly, we found that neither the size of the RRP nor the kinetics of its release was altered by the heterozygous STXBP1 mutation (Figure 5G). Thus, the heterozygous STXBP1 mutation decreased the magnitude of $\mathrm{Ca}^{2+}$ 
A

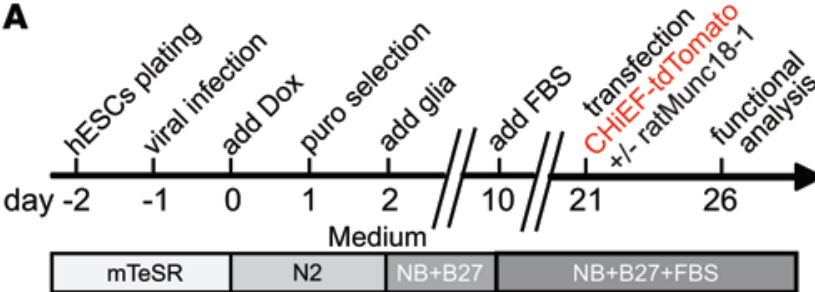

C

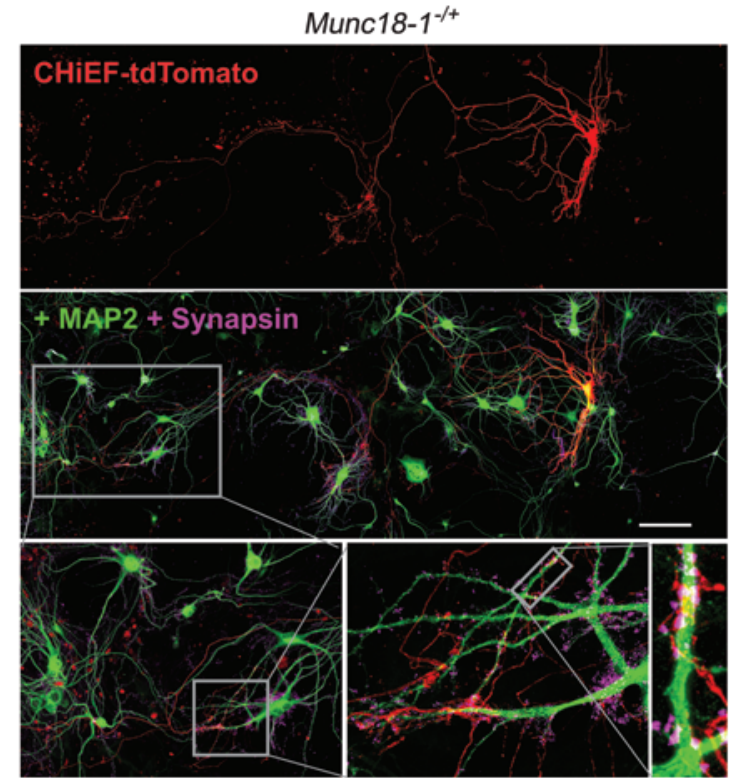

B

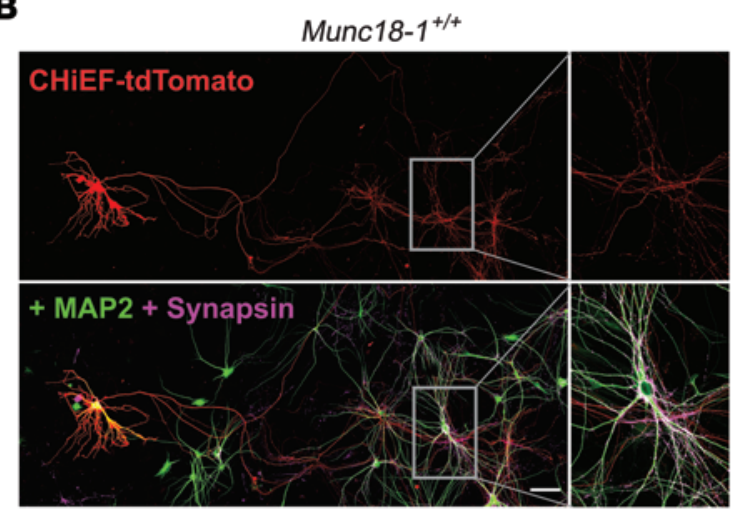

D

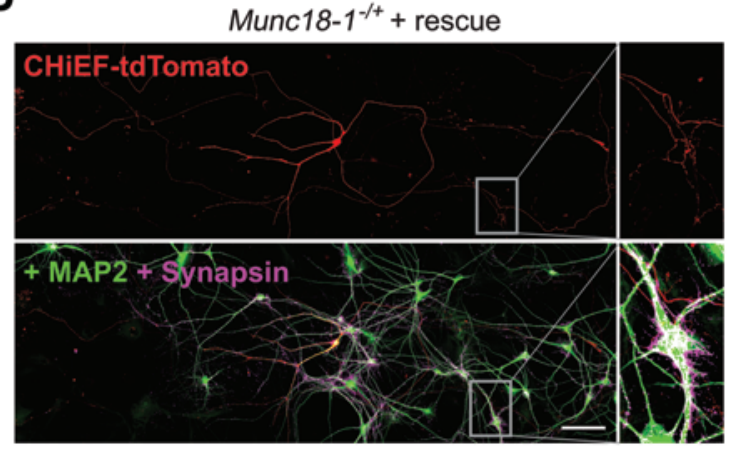

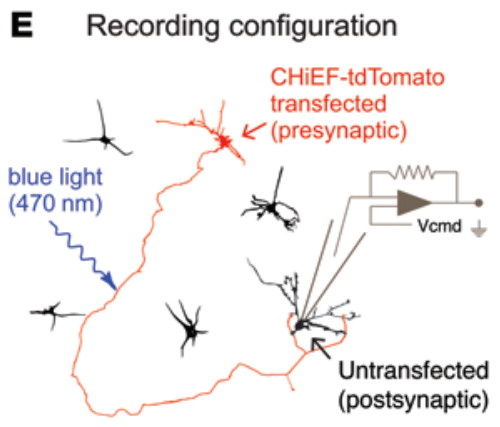

F Munc18-1 $1^{+/+}$

$2 \mathrm{~ms}$ light

$2 \mathrm{~ms}$ light

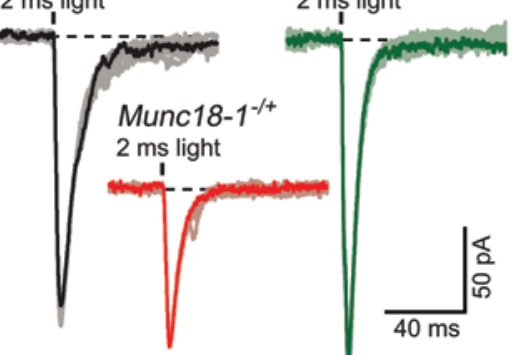

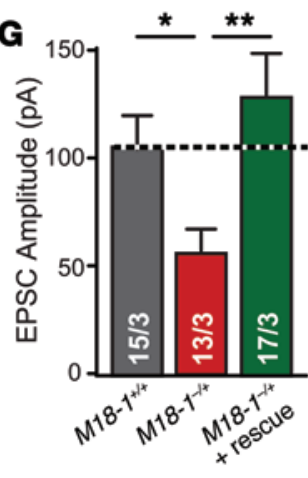

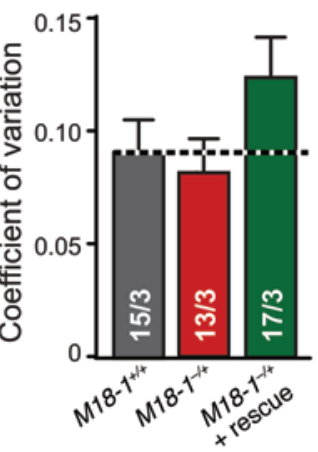

Figure 6. Heterozygous STXBP1 mutations decrease presynaptic neurotransmitter release at iN cell synapses as revealed by optogenetic analysis of unitary synaptic connections. (A) Flow diagram of optogenetic iN cell experiments using sparse channelrhodopsin transfections. Heterozygous STXBP1mutant or WT control iN cells were generated as described for Figure 1, sparsely transfected at day 21 with tdTomato-CHiEF (a derivative of channelrhodopsin-2), and analyzed by patch-clamping at day 26. For rescue experiments, rat Munc18-1 was cotransfected with channelrhodopsin at day 21. (B-D) Representative confocal micrographs of transfected iN cells expressing tdTomato-CHiEF (red); iN cells were counterstained for MAP2 (green) and synapsin (pink). Higher magnification images of the boxed areas are shown on the right (B1, WT control; B2, heterozygous STXBP1-mutant without rescue; B3, heterozygous STXBP1-mutant with rescue). Scale bars: $100 \mu \mathrm{m}$. (E) Schematic diagram of optogenetic analyses of unitary synaptic connections. tdTomatoCHiEF-positive presynaptic neurons were activated by short light pulses, and EPSCs were recorded from tdTomato-CHiEF-negative postsynaptic neurons. (F) Sample traces of light-evoked EPSCs. Black bar above the traces illustrates the 2-ms light pulse. (C) Summary graphs showing EPSC amplitudes (left) and their coefficient of variation (right). Graphs display mean \pm SEM; number of cells/independent cultures analyzed are indicated in the bars. ${ }^{*} P<0.05$; ${ }^{* *} P<0.01$, Student's $t$ test.

triggered neurotransmitter release without changing short-term plasticity, indicating an effect that operates downstream of vesicle priming into the RRP, but upstream of $\mathrm{Ca}^{2+}$ triggering of release.

To further define and independently confirm the surprisingly strong phenotype of heterozygous STXBP1-mutant human neurons, we used optogenetics to analyze unitary connections.
Since such experiments are not yet routine in the field, we developed and tested 2 different approaches for these analyses, both of which provided similar results (Figures 6 and 7).

In the first approach, we produced control and heterozygous STXBP1-mutant neurons as described above (Figure 1D), but sparsely transfected the neurons with a plasmid expressing a 
A

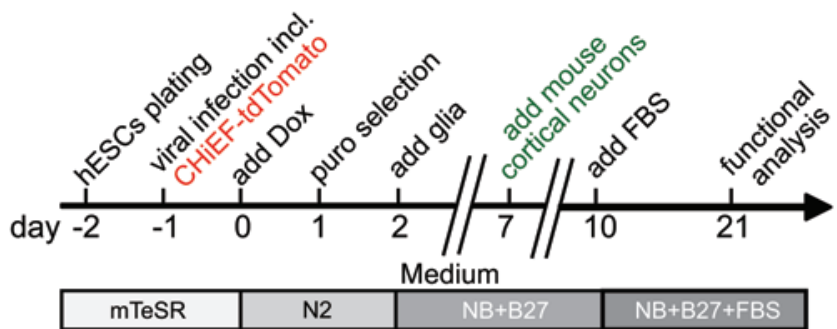

B

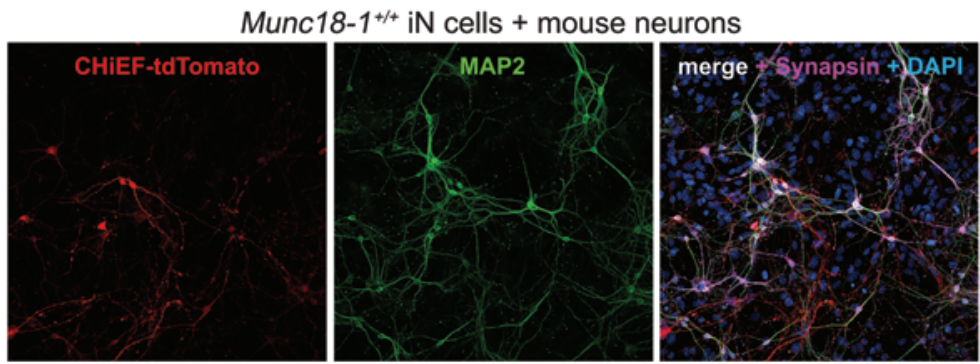

Munc18-1/1+ iN cells + mouse neurons
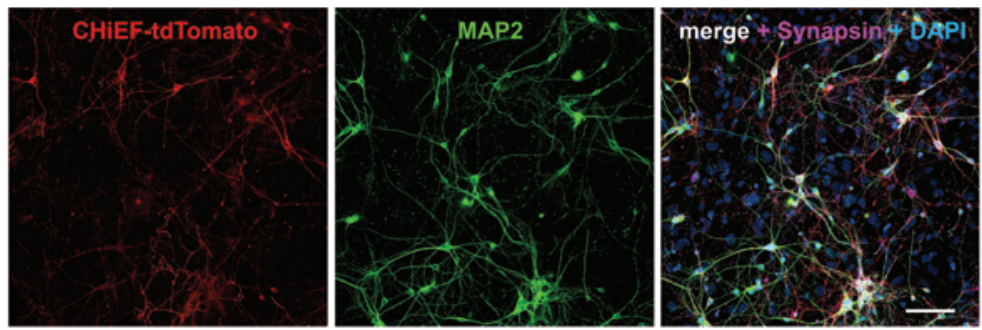

Figure 7. Heterozygous STXBP1 mutations decrease presynaptic neurotransmitter release at synapses formed by in cells onto cocultured mouse cortical neurons as revealed by optogenetic analysis of unitary synaptic connections. (A) Flow diagram of optogenetic iN cell experiments using cocultured mouse neurons. Heterozygous STXBP1-mutant or WT control neurons were generated as described for Figure 1, but with coexpression of tdTomato-CHiEF. iN cells were cocultured with a large excess of mouse cortical neurons at day 7 and analyzed by patchclamping at day 21. (B) Representative micrographs of tdTomato-CHiEF-transduced human neurons (red) that were cocultured with primary cortical mouse neurons on day 7 and analyzed on day 21 . All neurons were labeled with MAP2 (green), synapsin (pink), and DAPI (blue). Scale bar: $100 \mu \mathrm{m}$. (C) Sample traces of light-evoked EPSCs recorded from mouse neurons. (D) Summary graphs of the amplitude of optogenetically evoked EPSCs (left) and of the coefficient of variation (right). Summary graphs show mean $\pm \mathrm{SEM}$; numbers of cells/independent cultures analyzed are indicated in the bars. ${ }^{*} P<0.05 ;{ }^{*} P<0.01 ;{ }^{* *} P<0.001$, Student's $t$ test comparing heterozygous STXBP1 mutants to controls.
C

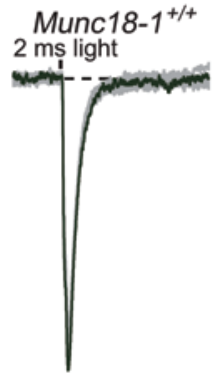

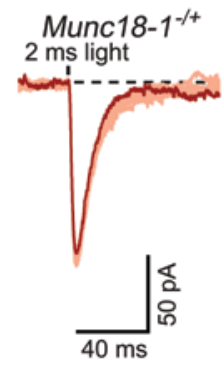

D

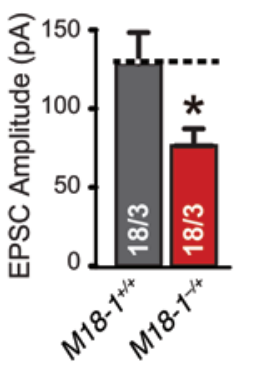

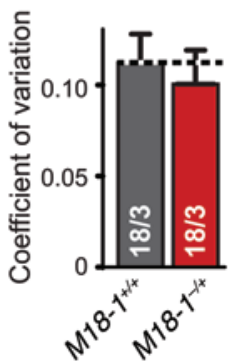

tdTomato-tagged and codon-optimized channelrhodopsin variant tdTomato-CHiEF (36), was expressed either alone or together with a Munc18-1 rescue construct. Transfections were performed at 21 days after induction of iN cell differentiation, and neurons were analyzed 5 days after the transfection by patching nontransfected neurons and measuring EPSCs induced by a 2-ms pulse of blue light (Figure 6A). Fluorescence microscopy confirmed that a small subset of neurons was transfected, but that these neurons formed abundant synapses on nontransfected neurons (Figure 6, B-D). With this experimental configuration, we were able to selectively optogenetically stimulate the inputs of 1 or possibly 2 presynaptic, channelrhodopsin-expressing neurons onto the patched postsynaptic target neuron and to measure the response to single-action potentials induced by a brief optical stimulation (Figure 6E). Moreover, by cotransfecting a plasmid expressing Munc18-1, this experimental configuration allows assessing the ability of WT Munc18-1 to rescue the presumptive phenotype. Note that the rescue is exclusively in the presynaptic neuron expressing the channelrhodopsin, whereas all other neurons are mutant, and that the rescue is introduced into the mutant presynaptic neurons after these neurons developed dendrites and axons and formed synapses.

Optogenetic stimulation of presynaptic neurons demonstrated that, compared with control neurons, heterozygous STXBP1-mutant neurons exhibited an approximately 2-fold decrease in EPSC amplitude (Figure 6, F and G). As expected for stimulation of only 1 and possibly 2 presynaptic neurons, the absolute EPSC amplitude produced by optogenetic stimulation of sparsely transfected control neurons was almost 10-fold smaller than that produced by electrical stimulation of all neurons (compare Figure 5B with Figure 6G). The optogenetically evoked EPSC amplitude corresponds to approximately $5 \mathrm{mEP}-$ SC amplitudes, while the electrically evoked EPSC corresponds to approximately 50 mEPSC amplitudes (compare Figure 4 with Figure 6E), suggesting that a single neuron forms between 6 and 10 synaptic contacts and that electrical stimulation activates about 10 times more synapses than optogenetic stimulation. 
Strikingly, presynaptic expression of WT Munc18-1 in STXBP1mutant neurons only 5 days before the recordings completely rescued the phenotype, demonstrating that the phenotype was due to a functional presynaptic impairment that could be reversed after development (Figure 6F). We also measured the coefficient of variation as an indirect assessment of the probability of $\mathrm{Ca}^{2+}$-triggered release, but observed no change in mutant neurons (Figure 6G), consistent with a lack of change in short-term plasticity (Figure 5).

In the second optogenetic approach, we coinfected conditionally STXBP1-mutant ES cells with an expression lentivirus for tdTomato-CHiEF at day -1 together with the lentiviruses that are used to induce directed differentiation of ES cells into neurons and with the lentiviruses that generate control WT or heterozygous STXBP1mutant neurons (Figure 7A). In this manner, all iN cells expressed the channelrhodopsin variant CHiEF. We then cocultured the iN cells on day 7 with an excess of primary cortical neurons freshly cultured from newborn mice, such that a sparse population of channelrhodopsin-expressing iN cells was mixed with more abundant WT mouse neurons (Figure 7B). Mouse neurons were patched after day 21, and synaptic transmission was stimulated optogenetically in presynaptic iN cells. We found that, as in the first optogenetic approach, the heterozygous STXBP1 mutation decreased the EPSC amplitude almost 2-fold, confirming the phenotype in this experimental protocol (Figure 7, C and D). Again, we observed no change in the coefficient of variation. Thus, with 2 distinct experimental approaches, we confirmed that the heterozygous $S T X B P 1$ mutation causes an approximately 2 -fold decrease in synaptic strength in the excitatory neurons produced by the iN cell protocol.

\section{Discussion}

In the present study, we investigated in human neurons whether heterozygous or homozygous STXBP1 loss-of-function mutations cause significant impairments in neuronal development, dendritic arborization, neuronal survival, and synaptic transmission. This project was motivated by the clinical observation that heterozygous mutations in the STXBP1 gene, which encodes the synaptic membrane-fusion protein Munc18-1, cause a severe form of infantile encephalopathy referred to as Ohtahara syndrome (20-22). Although hundreds of patients with STXBP1 mutations have been described, the pathogenetic mechanism of these mutations is unclear. In mice, it has been well established that homozygous deletion of STXBP1 produces a total block in synaptic transmission and neurodegeneration (31), but that the heterozygous deletion of $S T X B P 1$ induces only a mild phenotype $(37,38)$. Thus, the question arises of how a heterozygous STXBP1 mutation that in mice has only modest effects can cause severe clinical impairment in human patients. To address this question, we here examined the phenotype of heterozygous and homozygous STXBP1 mutations in human neurons, guided by the notion that human neurons may react differently to these mutations than mouse neurons. Indeed, we find that the heterozygous $S T X B P 1$ deletion is sufficient in human neurons for producing a major impairment in synaptic transmission, but does not cause a change in the development, dendritic arborization, or survival of the neurons. The homozygous STXBP1 deletion, in contrast, greatly decreased neuronal survival in a way that was similar to its effect on mouse neurons.
The phenotype of heterozygous STXBP1-mutant human neurons is sufficiently severe to plausibly account for the clinical presentation of Ohtahara syndrome patients, although additional effects of the STXBP1 mutation beyond those analyzed here may contribute to the clinical presentation. The nature of the phenotype we observed in heterozygous STXBP1-mutant human neurons is surprising in 2 respects. First, we found that a relatively small decrease in Munc18-1 with a concordant decrease in Syntaxin-1 levels caused a large decrease in neurotransmitter release. This result indicates that Munc18-1 and Syntaxin- 1 are subunits of an interdependent complex that is rate limiting for neurotransmitter release even when the overall levels of protein are only partially decreased. The phenotype is unexpectedly large for a heterozygous mutation when compared with the mouse phenotype $(31,32,37)$, possibly because human neurons are more sensitive to the loss of Munc18-1 than mouse neurons. Second, we found that the heterozygous STXBP1 mutation did not cause a decrease in the RRP or selectively affect only the initial release probability, but instead produced a uniform decrease in EPSC amplitudes throughout a stimulus train without a change in short-term plasticity. The decrease in mEPSC frequency without a change in mEPSC amplitude suggests that the decrease in EPSC amplitude reflects an impairment in $\mathrm{Ca}^{2+}$-triggered release, but the probability of $\mathrm{Ca}^{2+}$ triggering appears to be unchanged based on the unaltered short-term plasticity. We independently corroborated these conclusions using optogenetic approaches that analyzed synaptic transmission between pairs of neurons and that confirmed that the heterozygous Munc18-1 deletion caused a decrease in the strength of synapses but not in release probability, in this case as assessed by the coefficient of variation of the EPSC amplitude (Figures 6 and 7). The optogenetic experiments also demonstrated that the phenotype was produced by a purely presynaptic mechanism, since it could be rescued by presynaptic WT Munc18-1 (Figure 6) or could be induced by presynaptic deletion of the STXBP1 gene (Figure 7). Moreover, the optogenetic experiments showed that the STXBP1-mutant phenotype is not due to an impairment in neuronal development, but to a dysfunction of presynaptic terminals, as the phenotype could be rescued in neurons after neurites and synapses had formed (Figure 6). Thus, the decrease in the levels of the Munc18-1/Syntaxin-1 complex induced by the heterozygous STXBP1 mutation (Figure 2) appears to reduce the activity of vesicles downstream of priming but upstream of $\mathrm{Ca}^{2+}$ triggering of release. Independent of the mechanism that leads to this unusual phenotype, the observation of this phenotype and the approach in generating conditionally mutant human neurons and analyzing these neurons functionally as presented here could potentially be used to develop screening platforms for drugs that may reverse the decrease in release and may ameliorate the symptoms of patients with heterozygous $S T X B P 1$ mutations.

In the present experiments, we did not use integrating viral vectors for generating mutant ES cell clones, but generated conditional mutations that allowed comparison of mutant and WT neurons derived from the same clone (basically, after the mutations were conditionally induced, not a single cell division occurred). Moreover, we analyzed 2 independent mutant clones. Thus, the design of these experiments ensures that the observed phenotype is truly due to the intended mutation, as it was tightly controlled 
for genetic background, clonal variations, and genomic insertion effects (C.H. Pak, T. Danko, M. Wernig, and T.C. Südhof, unpublished observations). However, all of our experiments were performed based on one ES cell line, H1 cells, and we do not know how much the phenotype we observed was potentially influenced by the genetic background. In human patients, heterozygous STXBP1 mutations appear to invariably result in similar clinical presentations in contrast to many other human gene mutations that produce diverse phenotypes. This suggests that genetic background does not play a major role for the STXBP1 gene, probably because the encoded Munc18-1 performs a central function in membrane traffic, and we cannot at present assess how genetic background influences the phenotype. Nevertheless, the functional effects of the heterozygous STXBP1 mutation we here observe are profound. Since Munc18-1 is equally essential for neurotransmitter release in excitatory and inhibitory neurons $(31,39)$, these effects are probably also present in both, and both likely contribute to the clinical presentation in Ohtahara syndrome patients. The present data constitute what we believe is a first step toward understanding how the heterozygous STXBP1 mutation produces Ohtahara syndrome and suggest that the heterozygous mutation is indeed pathogenic, but additional steps will be required before potential therapeutic approaches can be considered.

\section{Methods}

Viral constructs. The following lentiviral constructs were used: (a) FUW-TetO-Ngn2-T2A-puromycin (where FUW indicates F-ubiquitin$\mathrm{W}$ ) expressing TetO-Ngn2-T2A-puromycin cassette (TetO promoter drives expression of full-length mouse Ngn2 and of puromycin via the cleavage-peptide sequence T2A; Figure 1C and ref. 29); (b) FUW-rtTA containing rtTA (29); (c) FUW-TetO-EGFP expressing EGFP (29); (d) FSW-NLS-mCherry expressing mCherry preceded by a nuclear localization sequence (under the control of neuron-specific human synapsin promoter) to monitor cell death; (e) FUW-GFP::Cre or FUWFlp to express Cre- or Flp-recombinase; (f) FUW-oCHiEF::tdTomato expressing the tdTomato-tagged channelrhodopsin variant oCHiEF. Two AAV constructs were used for gene targeting (also shown in Figure 1A). The first construct was as follows: for the targeting of the first allele of $S T X B P 1$ on chromosome 9, the construct contained sequences from the region encoding exon 2 flanked by loxP sites and an inverse orientated resistance cassette adjacent to the $5^{\prime}$ loxP site. The resistance cassette contained the PGK promotor, the puromycin resistance gene, and the SV40 polyA sequence. The resistance cassette was surrounded by frt sites. For homologous recombination, the $5^{\prime}$ arm of the construct included $1.5 \mathrm{~kb}$ of sequences located upstream of exon 2 . The $3^{\prime}$ arm contained $1.3 \mathrm{~kb}$ of sequences located downstream of exon 2. The second construct was as follows: for the targeting of the second allele, the sequence encoding the puromycin resistance gene of the first construct was exchanged with the blasticidin resistance gene. Everything else was unchanged.

Virus generation. Lentiviruses were produced as described (29) in HEK293T cells (ATCC) by cotransfection with 3 helper plasmids (pRSV$\mathrm{REV}, \mathrm{pMDLg} / \mathrm{pRRE}$, and vesicular stomatitis virus $\mathrm{G}$ protein expression vector) with $12 \mu \mathrm{g}$ of lentiviral vector DNA and $6 \mu \mathrm{g}$ of each of the helper plasmid DNAs per $75 \mathrm{~cm}^{2}$ culture area) using calcium phosphate. Lentiviruses were harvested in the medium 48 hours after transfection, pelleted by centrifugation $(49,000 \mathrm{~g}$ for 90 minutes), resuspended in MEM, aliquoted, and frozen at $-80^{\circ} \mathrm{C}$. Only virus preparations with more than $90 \%$ infection efficiency as assessed by EGFP expression or puromycin resistance were used for experiments. AAV-DJ (40) was used to deliver the targeting construct for generation of cKO cells. AAVDJ was produced in HEK293T cells by cotransfection of pHelper, pDJ, and AAV vector ( $8.5 \mu \mathrm{g}$ of DNA per $75 \mathrm{~cm}^{2}$ culture area) using calcium phosphate. Cells were harvested 72 hours after transfection in PBS/1 mM EDTA and following 1 freezing/thawing cycle. AAVs were collected from cytoplasm using Benzonase nuclease at a final concentration of 50 units $/ \mathrm{ml}$ at $37^{\circ} \mathrm{C}$ for 30 minutes. After clearing the suspension from cell debris by slow centrifugation (3,000 $g$ for 30 minutes), AAVs were isolated after fast centrifugation $(400,000 \mathrm{~g}$ for 120 minutes) in iodixanol (gradient from $15 \%-60 \%$ ) from the $40 \%$ layer and further concentrated using centricon concentrating tube (100,000 MWCO, Millipore UFC0910024) according to the manufacturer's suggested protocol.

Cell culture. Experiments were performed as described (29). H1 ES cells (WiCell Research Resources) were maintained as feeder-free cells in mTeSR1 medium (Stem Cell Technologies). Mouse glial cells were cultured from the forebrain of newborn WT CD1 mice (41). Briefly, newborn mouse forebrain homogenates were digested with papain and EDTA for 20 minutes, and cells were dissociated by harsh trituration to avoid growing of neurons and plated onto T75 flasks in DMEM supplemented with $10 \%$ FBS. Upon reaching confluence, glial cells were trypsinized and replated at a lower density a total of 2 to 3 times to remove potential trace amounts of mouse neurons before the glial cell cultures were used for coculture experiment with iN cells.

Gene targeting in ES cells. H1 ES cells were transduced by AAVs containing the first or the second construct (described above). The first construct was used to generate conditional heterozygous mutant ES cells. Selection drug was added and kept in mTeSR1 medium until 2 days after transduction. The surviving ES cells were allowed to grow into colonies and individually picked. Five correctly targeted colonies out of 91 colonies in total were confirmed by PCR screening using 2 sets of PCRs with oligosequences CATGTTAACCAGGATGGTCTCAATCT and ATAACTTCGTATAATGTATGCTATACGAAGTTAT or ATAACTTCGTATAGCATACATTATACGAAGTTAT and CAAGATCCCATCTCATAATA, spanning from the loxP site to outside of the targeting construct upstream or downstream of exon 2, respectively. The second construct was used to generate conditional homozygous mutant ES cells. Homologous recombination was achieved by transduction of the heterozygous clone no. 1 and subsequent drug selection with blasticidin and puromycin. Two correctly targeted colonies out of 80 colonies were confirmed by PCR screening using oligosequences GGGGGAATGGAAGGTGAGTAGAAAGTA and TAACTGCCTGACCAGGTGGTCTTTAAGA (referred to as primers no. 2 and no. 3 in Figure 1A). Successful excision of exon 2 upon Cre-recombinase-mediated recombination was checked for by using oligosequences: GGTGGGTTGGTTATGGCTCAGTAAAC and TAACTGCCTGACCAGGTGGTCTTTAAGA (referred to as primers no. 1 and no. 3 in Figure 1A).

Standard protocol for generation of iN cells from conditional mutant human ES cells and for activating the conditional mutations. iN cell generation has been described previously (29). Briefly, targeted human ES cells were treated with Accutase (Innovative Cell Technologies) and plated as dissociated cells in 24 -well plates $\left(1 \times 10^{4}\right.$ cells/well $)$ on day -2 (Figure 1B). Cells were plated on Matrigel-coated (BD Biosciences) coverslips in mTeSR1 containing $2 \mu \mathrm{M}$ thiazovivin (BioVision). On day -1 , lentiviruses prepared as described above $(0.3 \mu \mathrm{l} /$ well of 24 -well 
plate) were added in fresh mTeSR1 medium containing polybrene (8 $\mu \mathrm{g} / \mathrm{ml}$, Sigma-Aldrich). Two different types of lentiviruses were coinfected: the lentiviruses used for in cell induction, as described (29), and lentiviruses expressing either Flp-recombinase (to restore the WT gene) or Cre-recombinase (to create a null allele) under control of the ubiquitin promoter (Figure 1D). On day 0 , the culture medium was replaced with N2/DMEM/F12/NEAA (Invitrogen) containing human BDNF (10 ng/ml, PeproTech), human NT-3 (10 ng/ml, PeproTech), and mouse Laminin-1 $(0.2 \mu \mathrm{g} / \mathrm{ml}$, Invitrogen). Doxycycline $(2 \mu \mathrm{g} / \mathrm{ml}$, Clontech) was added on day 0 to induce TetO gene expression and retained in the medium until the end of the experiment. On day 1 , a 24 -hour puromycin selection $(1 \mu \mathrm{g} / \mathrm{ml})$ period was started. On day 2, mouse glia cells were added in neurobasal medium supplemented with B27/Glutamax (Invitrogen) containing BDNF, NT3, and Laminin-1; Ara-C (2 $\mu \mathrm{M}$, Sigma-Aldrich) was added to the medium to inhibit astrocyte proliferation. After day $2,50 \%$ of the medium in each well was exchanged every 2 days. FBS $(2.5 \%)$ was added to the culture medium on day 10 to support astrocyte viability, and iN cells were assayed after at least 21 days in most experiments.

Generation of $i N$ cells for optogenetic experiments. For the channelrhodopsin (tdTomato-CHiEF) transfection experiments (Figure 6), iN cells produced with the standard protocol were sparsely transfected by calcium phosphate on day 21 with a tdTomato-CHiEF expression vector without or with cotransfection with a Munc18-1 expression vector (FSW rat Munc18-1). Cells were analyzed at least 5 days later to ensure robust channelrhodopsin expression. For mouse coculture experiments (Figure 7), the standard protocol for iN cell production was modified by coinfection on day -1 with an additional lentivirus for tdTomatoCHiEF, and freshly dissected and dissociated primary mouse cortical cells were added on day 7. Cells were analyzed 14 days later (day 21).

Immunofluorescence and immunoblotting experiments. Immunofluorescence experiments were performed essentially as described (29). Briefly, cultured iN cells were fixed in $4 \%$ paraformaldehyde in PBS for 20 minutes at room temperature, washed 3 times with PBS, and incubated in $0.2 \%$ Triton X-100 in PBS for 10 minutes at room temperature. Cells were blocked in PBS containing $5 \%$ goat serum for 1 hour at room temperature. Primary antibodies were applied overnight at $4^{\circ} \mathrm{C}$, cells were washed in PBS 3 times, and fluorescent-labeled secondary antibodies (Alexa Fluor, 1:1000) were applied for 1 hour at room temperature. The following antibodies were used in immunocytochemistry experiments: MAP2 (Sigma-Aldrich; 1:1,000), Synapsin (E028; 1:2,000), Nanog (Millipore; 1:1000), Oct4 (sc-8628, Santa Cruz Biotechnology Inc.; 1:1000), SSEA-4 (Millipore; 1:1000), Tra-160 (Millipore; 1:1000), and Tra-1-81 (Millipore; 1:1000). Neuron dendritic morphology was visualized by MAP2 immunocytochemistry. All quantitative immunoblotting experiments were performed with iodinated $\left({ }^{125} \mathrm{I}\right)$ secondary antibodies as described (42). Samples were separated by SDS-PAGE and transferred onto nitrocellulose membranes. Blots were blocked in Tris-buffered saline containing 0.1\% Tween 20 (Sigma-Aldrich) and 5\% fat-free milk for 2 hours at room temperature. The blocked membrane was incubated in blocking buffer containing the primary antibody overnight at $4^{\circ} \mathrm{C}$, followed by 3 to 5 washes. The washed membrane was incubated in blocking buffer containing either HRP-conjugated secondary antibody (MP Biomedicals, 1:8000) for 2 hours at room temperature or ${ }^{125} \mathrm{I}$-labeled secondary antibody (PerkinElmer, 1:1000) overnight at room temperature. HRP immunoblots were developed with enhanced chemiluminescence (GE Healthcare).
${ }^{125}$ I blots were exposed to a phosphorimager screen (Amersham) for 1 to 7 days and scanned with a Storm scanner (GE Healthcare), followed by quantification with ImageQuant software (GE Healthcare). For immunodetection, the following antibodies were used: NeuN (ABN78, Millipore), TuJ1 (MMS-435P, Covance), complexin 1/2 (L668), Munc18 (610336, BD Transduction), SNAP25 (P913), synaptobrevin-2 (P939), synaptotagmin1 (41.1, Synaptic Systems), synapsin (E028), Syntaxin-1 (438B), $\beta$-actin (A1978, Sigma-Aldrich), Syntaxin-16 (4398), synaptophysin (Synaptic Systems, 7.2), GDP-dissociation inhibitor (Synaptic Systems, GDI) (81.2), vasolin-containing protein (Synaptic Systems, VCP), calmodulin-associated serine/threonine kinase (CASK, N3927), L1CAM (UJ127.11, Sigma-Aldrich), and SynCAM (T2412).

Gene-expression analyses. For quantitative real-time PCR (RT-PCR) analyses of pooled cultured cells, RNA was isolated using the RNAqueous Kit (Applied Biosystems), treated with DNase (Applied Biosystems), and reverse transcribed with Superscript III (Invitrogen). mRNA levels were quantified by RT-PCR assay using the Applied Biosystems 7900HT Fast Real-Time PCR system and RQ analysis software.

Quantification of morphology, synaptic density, and survival. Morphology and synaptic density analyses were essentially performed as described (43). Briefly, images were acquired using a Leica DFC400 digital camera, attached to a Leica DMIL LED inverted microscope with a $\times 10$ objective, driven by Leica Application Suite image-acquisition software. Images from 30 to 40 neurons per condition were reconstructed using the MetaMorph neurite application, scoring for total dendritic length, dendritic branch points, and soma area. For synapsin puncta analyses, images were acquired using a Nikon A1RSi confocal microscope system and the puncta density was determined using the software Nikon NIS-Elements. Analyses of the survival of iN cells were performed by 2 methods. First, survival was directly monitored using images of iN cell nuclei expressing mCherry at the same position of the culture dish taken every other day. The number of cells was determined using ImageJ software (http://imagej.nih. gov/ij/). For each experimental condition, the average of the pictures from 20 to 30 culture wells (of a 96-well culture plate) was considered as $n=1$. In total, the average of $n=3$ was calculated. Second, the total amount of genomic GAPDH gene DNA and of GAPDH mRNA in iN cells was monitored by quantitative PCR. DNA and RNA samples were collected from iN cells after 4 weeks of culture using DNeasy Blood \& Tissue Kit (QIAGEN; catalog 69504) or the RNAqueousMicro Kit (Ambion; catalog1931), respectively. DNA and RNA concentrations were measured by NanoDrop 1000 Spectrophotometer. Quantitative RT-PCRs were performed using USB VeriQuest Probe One-Step qRT-PCR Master Mix (Affymetrix; catalog 75700) on an Applied Biosystems 7900HT Fast Real-Time PCR system and RQ analysis software. RT-PCR was conducted following the manufacturer's suggested protocol: 1 cycle at $50^{\circ} \mathrm{C}$ for 15 minutes; 1 cycle at $95^{\circ} \mathrm{C}$ for 10 minutes; 35 cycles of $95^{\circ} \mathrm{C}$ for 15 seconds and $60^{\circ} \mathrm{C}$ for $30 \mathrm{sec}-$ onds. A mouse sequence-specific GAPDH PrimeTime Assay (Integrated DNA Technologies) was used as a cell number loading control for the iN culture by detecting glia cell numbers. A human sequencespecific GAPDH PrimeTime Assay (Integrated DNA Technologies) was used to detect the abundance of human GAPDH on both DNA and mRNA levels in control and mutant iN cells. Relative iN cell numbers were estimated by human GAPDH levels normalized to mouse GAPDH levels. Sequences of PrimeTime Assays were as follows: mouse GAPDH; probe: TGTTCCAGTATGACTCCACTCACGG; 
forward primer: GTGGCAAAGTGGAGATTGTTG; reverse primer: TTGACTGTGCCGTTGAATTTG; human GAPDH; probe: CAGCAAGAGCACAAGAGGAAGAGAGA; forward primer: AGGGTGGTGGACCTCAT; reverse primer: TGAGTGTGGCAGGGACT.

Standard electrophysiology experiments. In cultured iN cells, action potentials were recorded in the current-clamp whole-cell configuration at room temperature (pipette solution: $123 \mathrm{mM}$ K-gluconate, $10 \mathrm{mM}$ $\mathrm{KCl}, 1 \mathrm{mM} \mathrm{MgCl}$, 10 mM HEPES-KOH, pH 7.2, 1 mM EGTA, $0.1 \mathrm{mM}$ $\mathrm{CaCl}_{2}, 1 \mathrm{mM} \mathrm{K} \mathrm{K}_{2} \mathrm{ATP}, 0.2 \mathrm{mM} \mathrm{Na}_{4} \mathrm{GTP}$, and $4 \mathrm{mM}$ glucose). Membrane potentials were kept near $-65 \mathrm{mV}$, and step currents were injected to elicit action potentials with 20-pA increments. Synaptic transmission and whole-cell voltage-dependent currents were monitored in the whole-cell voltage-clamp mode (pipette solution: $120 \mathrm{mM} \mathrm{CsCl,} 5 \mathrm{mM}$ $\mathrm{NaCl}, 1 \mathrm{mM} \mathrm{MgCl}$, 10 mM HEPES-NaOH, pH 7.4, 10 mM EGTA, 3 mM MgATP, $0.3 \mathrm{mM}$ NaGTP, and $10 \mathrm{mM}$ QX-314). The bath solution in all experiments contained the following: $140 \mathrm{mM} \mathrm{NaCl}, 5 \mathrm{mM} \mathrm{KCl}, 2 \mathrm{mM}$ $\mathrm{CaCl}_{2}, 2 \mathrm{mM} \mathrm{MgCl}_{2}, 10 \mathrm{mM}$ HEPES-NaOH, pH 7.4, and $10 \mathrm{mM}$ glucose. Synaptic responses were measured as described previously (44). Evoked synaptic responses were triggered by 1-ms current injection through a local extracellular electrode (FHC concentric bipolar electrode, catalog CBAEC75) with a Model 2100 Isolated Pulse Stimulator (A-M Systems), and recorded in voltage clamp mode using Multiclamp 700B amplifier Clampex 10.4 Data Acquisition Software (Molecular Devices). Data were digitized at $10 \mathrm{kHz}$ with a $2-\mathrm{kHz}$ low-pass filter. Data were analyzed using Clampfit 10.4 (Axon Instruments). Stimulus artifacts for evoked synaptic responses were removed for graphic representation.

Optogenetic electrophysiology experiments. In experiments utilizing sparsely transfected iN cells expressing tdTomato-CHiEF, transfected cells were identified by florescence microscopy, and surrounding nonfluorescent iN cells visualized via DIC optics were patched. EPSCs were then measured as a function of 2-ms pulses of blue light $(470 \mathrm{~nm})$ generated with Lambda DG-4 light source (Sutter Instruments). An optic fiber connected to a dual-port condenser was used to guide the pulse into the microscope. The experiments using cocultured mouse cortical neurons were performed analogously, except that mouse neurons surrounding the human tdTomato-CHiEF-expressing iN cells were patched.

Data presentation and statistics. All data shown are mean $\pm \mathrm{SEM}$; all statistical analyses were performed using either 2-tailed Student's $t$ test (or otherwise indicated), 2-way ANOVA, or the KS test comparing the test sample with the control sample examined in the same experiments.

Study approval. The present study was approved by Stem Cell Research Oversight (SCRO) at Stanford University Research Compliance Office, Stanford University (SCRO 518: Studying brain diseases affecting synaptic transmission by using human induced neurons). Experiments involving animals were approved by the Stanford IACUC, Administrative Panel on Laboratory Animal Care (APLAC) Research Compliance Office, Stanford University.

\section{Acknowledgments}

This work was supported by grants from the NIH (MH092931 to M. Wernig, and AG010770 to T.C. Südhof) and from the California Institute for Regenerative Medicine (CIRM) (RT2 02061 to M. Wernig) and by a postdoctoral fellowship to C. Patzke (DFG PA 2110/1-1). M. Wernig is a New York Stem Cell Foundation-Robertson Investigator.

Address correspondence to: Christopher Patzke, Stanford University School of Medicine, 265 Campus Drive, Stanford, California 94305, USA. Phone: 650.724.5264; E-mail: patzke@stanford.edu.
1. State MW, Levitt P. The conundrums of understanding genetic risks for autism spectrum disorders. Nat Neurosci. 2011;14(12):1499-1506.

2. Malhotra D, Sebat J. CNVs: harbingers of a rare variant revolution in psychiatric genetics. Cell. 2012;148(6):1223-1241.

3. Huguet G, Ey E, Bourgeron T. The genetic landscapes of autism spectrum disorders. Annu Rev Genomics Hum Genet. 2013;14:191-213.

4. Devlin B, Scherer SW. Genetic architecture in autism spectrum disorder. Curr Opin Genet Dev. 2012;22(3):229-237.

5. Sullivan PF, Daly MJ, O’Donovan M. Genetic architectures of psychiatric disorders: the emerging picture and its implications. Nat Rev Genet. 2012;13(8):537-551.

6. Egawa N, et al. Drug screening for ALS using patient-specific induced pluripotent stem cells. Sci Transl Med. 2012;4(145):145ra104.

7. Dimos JT, et al. Induced pluripotent stem cells generated from patients with ALS can be differentiated into motor neurons. Science. 2008;321(5893):1218-1221.

8. Chamberlain SJ, Li XJ, Lalande M. Induced pluripotent stem (iPS) cells as in vitro models of human neurogenetic disorders. Neurogenetics. 2008;9(4):227-235.

9. Hargus G, et al. Differentiated Parkinson patientderived induced pluripotent stem cells grow in the adult rodent brain and reduce motor asymmetry in Parkinsonian rats. Proc Natl Acad Sci US A. 2010;107(36):15921-15926.

10. Soldner F, et al. Generation of isogenic pluripotent stem cells differing exclusively at two early onset Parkinson point mutations. Cell. 2011;146(2):318-331.

11. Brennand KJ, et al. Modelling schizophrenia using human induced pluripotent stem cells. Nature. 2011;473(7346):221-225.

12. Shi Y, Kirwan P, Smith J, MacLean G, Orkin SH, Livesey FJ. A human stem cell model of early Alzheimer's disease pathology in Down syndrome. Sci Transl Med. 2012;4(124):124ra129.

13. Ryan SD, et al. Isogenic human iPSC Parkinson's model shows nitrosative stress-induced dysfunction in MEF2-PGC1 $\alpha$ transcription. Cell. 2013;155(6):1351-1364.

14. Shcheglovitov A, et al. SHANK3 and IGF1 restore synaptic deficits in neurons from $22 \mathrm{q} 13$ deletion syndrome patients. Nature. 2013;503(7475):267-271.

15. Badger JL, Cordero-Llana O, Hartfield EM, Wade-Martins R. Parkinson's disease in a dish using stem cells as a molecular tool. Neuropharmacology. 2014;76(pt A):88-96.

16. Bundo $\mathrm{M}$, et al. Increased 11 retrotransposition in the neuronal genome in schizophrenia. Neuron. 2014;81(2):306-313.

17. Brennand K, et al. Phenotypic differences in hiPSC NPCs derived from patients with schizo- phrenia. Mol Psychiatry. 2015;20(3):361-368.

18. Marchetto MC, Gage FH. Your brain under the microscope: the promise of stem cells. Cerebrum. 2014;2014:1.

19. Soldner F, Jaenisch R. Medicine. iPSC disease modeling. Science. 2012;338(6111):1155-1156.

20. Saitsu H, et al. De novo mutations in the gene encoding STXBP1 (MUNC18-1) cause early infantile epileptic encephalopathy. Nat Genet. 2008;40(6):782-788.

21. Saitsu H, Kato M, Matsumoto N. Haploinsufficiency of STXBP1 and Ohtahara syndrome. In: Noebels JL, Avoli M, Rogawski MA, Olsen RW, DelgadoEscueta AV, eds. Jasper's Basic Mechanisms of the Epilepsies. 4th ed. Bethesda, Maryland, USA: 2012

22. Pavone P, Spalice A, Polizzi A, Parisi P, Ruggieri M. Ohtahara syndrome with emphasis on recent genetic discovery. Brain Dev. 2012;34(6):459-468.

23. Milh M, et al. Epileptic and nonepileptic features in patients with early onset epileptic encephalopathy and STXBP1 mutations. Epilepsia. 2011;52(10):1828-1834.

24. Hamdan FF, et al. Intellectual disability without epilepsy associated with STXBP1 disruption. Eur JHum Genet. 2011;19(5):607-609.

25. Mignot C, et al. STXBP1-related encephalopathy presenting as infantile spasms and generalized tremor in three patients. Epilepsia. 2011;52(10):1820-1827.

26. Hong W, Lev S. Tethering the assembly of SNARE 
complexes. Trends Cell Biol. 2014;24(1):35-43.

27. Rizo J, Sudhof TC. The membrane fusion enigma: SNAREs, Sec1/Munc18 proteins, and their accomplices - guilty as charged? Annu Rev Cell Dev Biol. 2012;28:279-308.

28. Pak $\mathrm{CH}$, et al. Human neuropsychiatric disease modeling using conditional deletion reveals synaptic transmission defects caused by heterozygous mutations in NRXN1. Cell Stem Cell. In press.

29. Zhang Y, et al. Rapid single-step induction of functional neurons from human pluripotent stem cells. Neuron. 2013;78(5):785-798.

30. Pang ZP, et al. Induction of human neuronal cells by defined transcription factors. Nature. 2011;476(7359):220-223.

31. Verhage $M$, et al. Synaptic assembly of the brain in the absence of neurotransmitter secretion. Science. 2000;287(5454):864-869.

32. Toonen RF, de Vries KJ, Zalm R, Sudhof TC, Verhage M. Munc18-1 stabilizes syntaxin 1, but is not essential for syntaxin 1 targeting and SNARE complex formation. J Neurochem. 2005;93(6):1393-1400.
33. Gerber SH, et al. Conformational switch of syntaxin-1 controls synaptic vesicle fusion. Science. 2008;321(5895):1507-1510.

34. Zhou P, Bacaj T, Yang X, Pang ZP, Sudhof TC. Lipidanchored SNAREs lacking transmembrane regions fully support membrane fusion during neurotransmitter release. Neuron. 2013;80(2):470-483.

35. Rosenmund C, Stevens CF. Definition of the readily releasable pool of vesicles at hippocampal synapses. Neuron. 1996;16(6):1197-1207.

36. Lin JY, Lin MZ, Steinbach P, Tsien RY. Characterization of engineered channelrhodopsin variants with improved properties and kinetics. Biophys J. 2009;96(5):1803-1814.

37. Hager T, Maroteaux G, Pont P, Julsing J, van Vliet $\mathrm{R}$, Stiedl O. Munc18-1 haploinsufficiency results in enhanced anxiety-like behavior as determined by heart rate responses in mice. Behav Brain Res. 2014;260:44-52.

38. Toonen RF, et al. Munc18-1 expression levels control synapse recovery by regulating readily releasable pool size. Proc Natl Acad Sci U S A. 2006;103(48):18332-18337.
39. Deak F, et al. Munc18-1 binding to the neuronal SNARE complex controls synaptic vesicle priming. J Cell Biol. 2009;184(5):751-764.

40. Grimm D, et al. In vitro and in vivo gene therapy vector evolution via multispecies interbreeding and retargeting of adeno-associated viruses. JVirol. 2008;82(12):5887-5911.

41. Franke B, Figiel M, Engele J. CNS glia are targets for GDNF and neurturin. Histochem Cell Biol. 1998;110(6):595-601.

42. Rosahl TW, et al. Essential functions of synapsins I and II in synaptic vesicle regulation. Nature. 1995;375(6531):488-493.

43. Anderson GR, Galfin T, Xu W, Aoto J, Malenka $\mathrm{RC}$, Sudhof TC. Candidate autism gene screen identifies critical role for cell-adhesion molecule CASPR 2 in dendritic arborization and spine development. Proc Natl Acad Sci U S A. 2012;109(44):18120-18125.

44. Maximov A, Pang ZP, Tervo DG, Sudhof TC. Monitoring synaptic transmission in primary neuronal cultures using local extracellular stimulation. J Neurosci Methods. 2007;161(1):75-87. 